\title{
Reducing Attrition in Physical Activity Programs for Older Adults
}

\author{
Jonine Jancey, Andy Lee, Peter Howat, \\ Ann Clarke, Kui Wang, and Trevor Shilton
}

\begin{abstract}
This study investigated attrition in a 6-month physical activity intervention for older adults. The program was based on the social-cognitive theory incorporating self-efficacy factors. Two hundred forty-eight insufficiently active 65- to 74-yearolds were recruited from the Australian federal electoral roll. The intervention comprised walking and strength and flexibility exercises and was conducted in 30 local neighborhoods where the participants resided. Characteristics of individuals lost to attrition $(\mathrm{n}=86,35 \%)$ were compared with those of program completers $(n=162,65 \%)$. Logistic-regression analysis showed that those lost to attrition came from areas of lower socioeconomic status, were overweight and less physically active, and had lower walking self-efficacy scores and higher loneliness scores. The results suggest that early assessment of these characteristics should be undertaken to identify individuals at risk of attrition, to improve retention, and to avoid potential bias.
\end{abstract}

Key Words: intervention, older people,

It is estimated half of all physical decline associated with aging is preventable if adequate levels of physical activity are maintained (O'Brien Cousins, 2003). Industrialized societies seem to disregard this compelling evidence, however, and continue to be attracted to an inactive lifestyle, especially in the older age groups (Bauman, Bellew, Vita, Brown, \& Owen, 2002; Resnick, 2001). This increase in inactivity among older people is associated with potential adverse health affects (Anderson, Schnohr, Schroll, \& Hein, 2000; Crespo et al., 2002; Lee \& Skerrett, 2000; Wannamethee, Shaper, \& Walker, 2000) and has resulted in an increased focus on encouraging and maintaining physical activity in the burgeoning older population.

Studies attempting to identify and understand factors associated with physical activity have been limited, especially with respect to longitudinal data (Wanzhu, Stump, Damush, \& Clark, 2004). Intervention programs aimed at mobilizing older populations and understanding associated factors have been variable, with reported attrition rates ranging from $22 \%$ to $76 \%$ within the first year of starting a new program (Prohaska, Peters, \& Warren, 2000; Schmidt, Gruman, King, \&

Jancey, Lee, Howat, Clarke, and Wang are with the School of Public Health, Curtin University of Technology, Western Australia Perth 6845. Shilton is with the National Heart Foundation. 
Wolfson, 2000) and the greatest number of dropouts occurring in the first 3 months (Schmidt et al.). In previous studies of sedentary older age groups, approximately $50 \%$ drop out within the first 6 months of involvement (Dishman, 1994; Ettinger et al., 1997; Resnick \& Spellbring, 2000), suggesting that older adults are particularly susceptible to exercise attrition (Prohaska et al.).

The social-cognitive approach to understanding physical activity of older adults provided the conceptual framework for this Perth Active Living Seniors (PALS) study. Social-cognitive theory (SCT) defines human behavior as a dynamic interaction of personal factors, behavior, and the environment, although all forces are not necessarily of equal strength (Bandura, 1986). From the SCT perspective, people are not driven solely by inner forces and are not automatically shaped or controlled by external influences. Rather, human behavior can be explained by reciprocal determinism, which involves human behavior, cognitive and other personal factors, and environmental events all operating as interacting determinants of one another (Bandura, 1997). SCT hypothesized that all behavioral changes are mediated by the cognitive mechanism termed self-efficacy and suggested that it is behavior specific (Bandura, 1986, 1997).

Self-efficacy refers to an individual's belief in his or her capabilities to carry out a specific course of action and has been identified as an important predictor of exercise adoption and maintenance (Bandura, 1986). Self-efficacy is theorized to influence one's choice of activity, the effort expended, and persistence (Bandura, 1986; McAuley \& Mihalko, 2003; Resnick \& Spellbring, 2000). It is recognized to decrease with age (Resnick, Palmer, Jenkins, \& Spellbring, 2000) and links to personal well-being (Bandura, 1997). According to SCT, successful participation in regular exercise should serve to enhance the perception of physical capabilities, consequently enhancing psychological and emotional well-being (Mihalko $\&$ McAuley, 1996; Treasure \& Newberry, 1998). On the other hand, a person's mood might influence his or her perception of self-efficacy, with a low mood disturbance and better overall mental health being associated with greater self-efficacy (Resnick, 2001).

According to King et al. (1992), factors affecting program attrition can be divided into three categories: environmental factors, program factors, and personbased factors. Person-based factors include demographics and individual perception and beliefs about exercise. Environmental factors include both the physical and social environment, such as social support and where the program is located. Program factors encompass program design, recruitment processes, and inclusion and exclusion criteria.

Although the design of a program (Mills et al., 2001) and, to a certain extent, the physical environment in which the program is set can be controlled by the investigators, serious consideration during the planning phase is required (Preloran, Browner, $\&$ Lieber, 2001) to ensure that attrition is minimized. The intervention program should be easy to access, with the cost being within reach of participants (Corti et al., 1995; Godin, Desharnais, Valois, Jobin, \& Bradet, 1994; Souder, 1992). The participants need to understand the program (Souder), and the program organizers need to understand the commitments of participants. In addition, the program's frequency, intensity, and duration should be appropriate for the participants, with friendly instructors, consistency of instruction, and peer support provided (Corti et al.; Dishman, 1994; Warren-Findlow, Prohaska, \& Freedman, 2003). 
The social environment, such as friends and family, has been consistently found to influence participation in physical activity (Chogahara, O'Brien Cousins, \& Wankel, 1998; Clark, 1999; Sallis, Bauman, \& Pratt, 1998; Stahl et al., 2001). Older people, however, generally receive less encouragement from others regarding their exercise habits (Hayslip, Weigand, Weinberg, Richardson, \& Jackson, 1996). This is of particular concern, because support might be more important to older adults (Chogahara et al.). There is also evidence suggesting that the reception and effect of social support are mediated by one's degree of self-efficacy (McAuley, Jerome, Elavsky, Marquez, \& Ramsey, 2003). Social support and inculcated values have been shown to be significant predictors of exercise in later life (O'Brien Cousins, 2003).

Person-based determinants associated with physical activity are broad and varied for older adults. For example, overweight adults are less likely to stay with a vigorous physical activity program (Dishman, 1991), and lower education levels have been linked to attrition (Jacomb, Jorm, Korten, Christensen, \& Henderson, 2002; Van Beijsterveldt et al., 2002), along with being older (Chatfield, Brayne, \& Matthews, 2005; Jacomb et al., 2002; Van Beijsterveldt et al.) and being separated from one's spouse (Garcia \& King, 1991; Martin \& Sinden, 2001). Conversely, demographics are found to be inconsistent predictors of adherence (Martin \& Sinden). Other reported determinants of attrition are fear of injury and illness (King, Rejeski, \& Buchner, 1998), low mental health score (Chatfield et al.; Jacomb et al.; Van Beijsterveldt et al.), being less healthy (Chatfield et al.; Van Beijsterveldt et al.), having lower social participation (Chatfield et al.), living alone (Chatfield et al.; Wilson \& Webber, 1976), and depression (Martin \& Sinden). A review of over 20 randomized controlled trials found that being fitter, having a history of being active, and high exercise self-efficacy were associated with greater adherence (Martin \& Sinden). The effect of prior exercise behavior on subsequent exercise behavior has been disregarded, even though it might be a main source of self-efficacy information (McAuley et al., 2003).

Loss of participants from research projects is a serious concern because it can potentially introduce bias and render a study less representative over time. When poor compliance affects statistical power, additional methods are required to recruit participants that can be costly and time-consuming (Martin \& Sinden, 2001). Keeping older people in physical-activity-intervention programs can contribute to the understanding of exercise behavior. Moreover, physical activity is seen as an economically attractive health-management approach for the aging population (Wanzhu et al., 2004).

Minimizing attrition is known to be critical in physical activity research involving older adults. The PALS study aimed to investigate this issue by identifying factors associated with nonadherence in a neighborhood-based physical activity intervention. It was anticipated that the outcomes would contribute to an improved understanding of older adults' physical activity behavior.

\section{Methods}

\section{Neighborhood Selection}

Following a stratified quasi-random sampling frame, 60 neighborhoods were selected from a pool of 388 neighborhoods in the greater metropolitan area of Perth, 
the capital of Western Australia, a city with a population of 1.4 million people. Selection criteria were (a) the neighborhood was composed of at least $11 \%$ of older adults 65 years of age or older, reflecting the state average (Australian Bureau of Statistics, 2001); (b) the neighborhood contained at least 100 people age $\geq 65$ years, to ensure a large enough sample size for matching of telephone numbers to the electronic white pages and; (c) the neighborhood was at least $1 \mathrm{~km}$ away from waterways, namely a river or beach, to minimize "coastal effects" on physical activity (Bauman, Smith, Stoker, \& Booth, 1999). Distance was calculated via a geographical information system. Using the socioeconomic index for area (SEIFA; Australian Bureau of Statistics, 1996), a value derived from income, educational attainment, employment status, and skill level, neighborhoods were arbitrarily matched for low, medium, and high levels of socioeconomic status. The neighborhoods were then assigned to either the intervention group or the control group of 30 neighborhoods each using a table of random numbers.

\section{Participant Selection}

Permission was sought from the Australian Electoral Commission for access to the federal electoral roll, which provides a comprehensive pool of potential participants, as all Australian citizens must be registered. From the roll approximately 120 participants per neighborhood were randomly selected. Equal numbers of 65- to 69-year-olds and 70- to 74-year-olds were generated. Based on a pilot study, this would enable the securing of a sample of 12-15 participants per neighborhood. The pool of potential participants was then matched to the Perth electronic white pages (a directory of addresses and telephone numbers) by a survey center specializing in health research.

\section{Recruitment Procedure}

Potential participants were sent postcards explaining that their names had been randomly selected for potential involvement in a health project that would consist of either a 6-month walking program or the completion of three questionnaires. The postcard detailed who was conducting the research and that a researcher might call within the next 2 weeks. Over a 2 -week period, trained recruiters telephoned potential participants and established eligibility. Prospective participants were required to meet the following criteria: be age 65-74 years and be healthy in the sense that participation in a low-stress walking program would not place them at risk or exacerbate any existing health condition. A maximum of six callbacks were made. The participants were randomly assigned to the intervention and control groups. Written consent was then sought from each participant. Ethical approval was obtained from the human research ethics committee of the researchers' institution.

\section{Survey Instrument}

The PALS questionnaire included previously validated instruments. The composite instrument was assessed for face and content validity by an expert panel and two focus groups representative of the target population. A test-retest study was conducted on a sample of 75 participants age 65-74 years over 2 weeks to determine reliability of the questionnaire. Intraclass correlation coefficients (ICCs) and 
paired-sample $t$ tests were used to examine correlations and differences of means, respectively, between the first and second survey.

The International Physical Activity Questionnaire (IPAQ) has undergone extensive reliability and validity testing and has acceptable measurement properties for use in population studies of physical activity participation (Craig et al., 2003). The instrument measured frequency and duration of walking for recreation, walking for transport, and other moderate physical activity. A moderate level of physical activity was chosen because responses from focus-group participants indicated a preference to avoid the sensation of a fast-beating heart. An explanation of moderate activity preceded the question, which consisted of two parts: "In a usual week how many times do you do moderate physical activity for at least $10 \mathrm{~min}$, such as swimming, dancing, or cycling?" and "In a usual week what do you estimate is the total time you spend doing these activities?" For the IPAQ, overall the ICCs were moderate to high (.58-.94), and no significant differences were found between the first- and second-survey results ( $t$-test $p$ ranged from .051 to .595 ), further supporting the reproducibility of the questionnaire. Levels of physical activity were divided into sufficient and insufficient, with sufficient physical activity being defined as achieving $30 \mathrm{~min}$ of moderate physical activity on at least 5 days per week, according to Australia's National Physical Activity Guidelines (Australia Department of Health and Aged Care, 1999).

Walking self-efficacy refers to measures directed at assessing an individual's belief regarding his or her capability to successfully complete incremental bouts of physical activity (McAuley \& Mihalko, 2003). The scale consisted of six items measuring the degree of confidence to perform walking over increasing periods of time (in minutes) on a Likert scale of 1 to 10 . The scores were summed and divided by the number of questions, with a higher score indicating greater walking efficacy. Our test-retest showed that walking self-efficacy was a highly reliable measure (ICC .85, $t$-test $p$.084).

The UCLA loneliness scale is an instrument commonly used across a variety of populations, including older people and in mail surveys (Russell, 1996). It measures subjective feelings of loneliness. It draws on the concept of loneliness being unidimensional in structure, assessing both the frequency and intensity of salient aspects and events of the lonely experience. The instrument consists of 20 items. The scale ranges from never to always, with a higher total score indicating more loneliness (ICC .72, $t$-test $p$.576).

The SEIFA (Australian Bureau of Statistics, 1996) is related to socioeconomic aspects of geographic area. The index is obtained by summarizing social and economic characteristics of families and households. It is derived from income, educational attainment, employment status, and skill level.

Self-reported body-mass index (BMI) is calculated as weight in kilograms divided by height in meters squared (Cameron et al., 2003). A BMI of 18.5-24.9 kg/ $\mathrm{m}^{2}$ was classified as normal weight, $25.0-29.9 \mathrm{~kg} / \mathrm{m}^{2}$ was classified as overweight, and BMI exceeding $30.0 \mathrm{~kg} / \mathrm{m}^{2}$ was classified as obese. Demographic information sought included gender, age, education level, relationship status, country of birth, and perceived financial struggle. Finally, the participants were asked the qualitative questions "What have you liked the most about the PALS project?" and "What do you think we could do to improve the PALS project?" 


\section{Intervention}

The prescriptive walking intervention, designed by an exercise gerontologist, commenced at a very low level. The intervention was conducted over a period of 6 months, providing a graduated program twice per week for insufficiently active older adults. The program contained aerobic activities, as well as balance, strength, and flexibility components. It was free of charge, locally based for easy access, and conducted in attractive and safe environments, thus enhancing a sense of community and safety. Trained walk leaders offered expert advice, reassurance, encouragement, feedback, and education in a range of health areas. They also monitored nonattendance and provided a critical link between researchers and participants. All participants received nonmonetary incentives such as small gifts, a walker's newsletter, and attractive educational material. Enhancement of social support, such as organized social gatherings, was a planned component of the program, which aimed to reduce the attrition rate of participants.

\section{Statistical Analysis}

All data were screened and outlying entries were confirmed and deleted before statistical analysis. Univariate statistics including chi-square and $t$ tests were applied to the intervention-group data, followed by logistic-regression analysis to determine factors affecting attrition of the walking program. Significance levels of 5\% and $1 \%$ were used for statistical tests. All statistical analyses were undertaken using the SPSS for Windows ${ }^{\circledR}$ package, version 11.

\section{Exit Interviews}

A sample of 16 participants (8 dropouts, 8 completers) was purposely chosen from randomly selected neighborhoods in the intervention group to participate in exit interviews. A researcher with experience in interviews and data transcription contacted these participants and sought their approval for the exit interview. After informed consent was obtained, an interview time was arranged, either at the participant's home or at a convenient location. All interviews were conducted and tape-recorded by the same researcher. Each interview took about $1 \mathrm{hr}$ and focused on reasons for dropping out of the PALS program, how the participant felt about attrition, and how the program could be more appealing. The relevant questions asked are provided in appendix A. Verbatim transcriptions were typed for each interview. Data were analyzed using a thematic-analysis approach in order to explore the dialogue for reasons for attrition.

\section{Results}

When potential participants were contacted to determine their willingness to be involved in the program, the initial refusal rate for participation was $87.9 \%$ (248 recruited out of 2,056 contacted) for the intervention group and $85.5 \%$ (313 recruited out of 2,153 contacted) for the control group. The intervention group included 248 participants, with 162 completing the program and 86 dropping out. This attrition rate of $34.7 \%$ was significantly higher $(p<.01)$ than the $31.9 \%$ reported 
in the control group. Of those that dropped out of the intervention group, most of the attrition occurred within the first 3 months $(n=66,77 \%)$. Table 1 provides sample descriptive statistics and compares the attrition rates for selected variables of interest in the intervention group. Most of the intervention-group participants were female $(66 \%)$ and highly educated $(30 \%)$. The dropouts and completers were similar in demographics, namely, age, gender, education level, relationship status, and country of birth, but significant differences in attrition rates were found with respect to SEIFA $(p<.05)$, BMI $(p<.05)$, and physical activity level $(p<.01)$. The mean walking-self-efficacy scores of dropouts $(7.45, S D=2.35)$ and completers $(8.70, S D=1.57)$ were significantly different according to a two-sample $t$ test $(p<.01)$. The mean UCLA loneliness score of dropouts $(40.12, S D=9.59)$ was also significantly higher $(p<.01)$ than that of completers $(36.58, S D=8.87)$.

Table 2 presents logistic-regression results of factors affecting attrition that were measured at baseline. The findings confirm those of the univariate analysis. Accounting for age and gender of the participants, the adjusted odds ratios of medium and high SEIFA indexes were 0.74 and 0.40 , respectively. In particular,

Table 1 Sample Characteristics and Attrition Rates, $n(\%)$

\begin{tabular}{|c|c|c|c|}
\hline Characteristic & $\begin{array}{l}\text { Dropouts, } \\
\quad n=86\end{array}$ & $\begin{array}{l}\text { All walkers, } \\
\qquad N=248\end{array}$ & $\begin{array}{l}\text { Attrition } \\
\text { rate }\end{array}$ \\
\hline \multicolumn{4}{|l|}{ Age } \\
\hline 66-69 years & $45(52 \%)$ & $122(49 \%)$ & $37 \%$ \\
\hline$\geq 70$ years & $41(48 \%)$ & $126(51 \%)$ & $33 \%$ \\
\hline \multicolumn{4}{|l|}{ Gender } \\
\hline female & $56(65 \%)$ & $163(66 \%)$ & $34 \%$ \\
\hline male & $30(35 \%)$ & $85(34 \%)$ & $35 \%$ \\
\hline \multicolumn{4}{|l|}{ Education level } \\
\hline primary & $14(16 \%)$ & $32(13 \%)$ & $43 \%$ \\
\hline secondary & $47(55 \%)$ & $141(57 \%)$ & $33 \%$ \\
\hline tertiary & $25(29 \%)$ & $75(30 \%)$ & $33 \%$ \\
\hline \multicolumn{4}{|l|}{ Relationship status } \\
\hline partner & $53(62 \%)$ & $166(67 \%)$ & $32 \%$ \\
\hline no partner & $33(38 \%)$ & $82(33 \%)$ & $40 \%$ \\
\hline \multicolumn{4}{|l|}{ Country of birth } \\
\hline Australian born & $54(63 \%)$ & $166(67 \%)$ & $33 \%$ \\
\hline non-Australian born & $32(37 \%)$ & $82(33 \%)$ & $39 \%$ \\
\hline \multicolumn{4}{|c|}{ Socioeconomic index for area* } \\
\hline & $36(42 \%)$ & $82(33 \%)$ & $44 \%$ \\
\hline medium & $29(34 \%)$ & $79(32 \%)$ & $37 \%$ \\
\hline high & $21(24 \%)$ & $87(35 \%)$ & $24 \%$ \\
\hline \multicolumn{4}{|l|}{ Body-mass index* } \\
\hline normal & $12(14 \%)$ & $58(23 \%)$ & $21 \%$ \\
\hline overweight & $42(49 \%)$ & $111(45 \%)$ & $38 \%$ \\
\hline obese & $32(37 \%)$ & $79(32 \%)$ & $41 \%$ \\
\hline \multicolumn{4}{|l|}{ Physical activity** } \\
\hline insufficient & $61(71 \%)$ & $134(54 \%)$ & $46 \%$ \\
\hline sufficient & $25(29 \%)$ & $114(46 \%)$ & $22 \%$ \\
\hline
\end{tabular}

${ }^{*} p<.05 .{ }^{* *} p<.01$. 
Table 2 Logistic-Regression Results of Factors Affecting Attrition $(N=248)$

\begin{tabular}{lcc}
\hline Factor & Odds ratio & $95 \%$ confidence interval \\
\hline $\begin{array}{l}\text { Age } \\
\quad 66-69 \text { years }\end{array}$ & 1 & \\
$\quad \geq 70$ years & 1.21 & $(0.66,2.19)$ \\
$\begin{array}{l}\text { Gender } \\
\quad \text { female } \\
\text { male }\end{array}$ & 1 & - \\
$\begin{array}{l}\text { Socioeconomic index for area } \\
\text { low } \\
\text { medium }\end{array}$ & 1.19 & $(0.63,2.23)$ \\
$\quad$ high* & 1 & \\
Body-mass index & 0.74 & $(0.37,1.47)$ \\
$\quad$ normal & 0.40 & $(0.19,0.83)$ \\
$\quad$ overweight* & 1 & \\
Phese & 2.29 & $(1.01,5.19)$ \\
$\quad$ sufficient & 1.88 & $(0.79,4.45)$ \\
$\quad$ insufficient** & 1 & $(1.30,4.43)$ \\
Walking self-efficacy** & 2.40 & $(0.66,0.89)$ \\
Loneliness* & 0.77 & $(1.01,1.07)$ \\
\hline
\end{tabular}

${ }^{*} p<.05 .{ }^{* *} p<.01$.

older adults residing in high-socioeconomic neighborhoods were less likely to drop out than those from low-socioeconomic areas $(p<.05)$. Conversely, the risk of dropout was about twice for overweight people than that of their normal-weight counterparts $(p<.05)$. Similarly, older adults who reported insufficient physical activity had a higher likelihood of dropping out of the program $(p<.01)$. The two continuous variables, walking self-efficacy and the UCLA loneliness score, were also significantly related to attrition. Participants with greater walking self-efficacy had better adherence to the walking program $(p<.01)$, whereas those who felt lonely, as reflected by a high loneliness score, appeared to have a significantly higher risk of attrition $(p<.05)$.

Of the walkers who responded to the qualitative questions, most believed that the group provided motivation to exercise $(n=73)$, enjoyed the companionship of other walkers $(n=64)$, and liked their walk leader $(n=43)$. They agreed that their walk leader was "encouraging" (99.5\%), "provided helpful guidance" (99\%), and "kept them going to the group" (88\%). Only 3.4\% disagreed that their walk leader "influenced their participation." With regard to improvement to the program, respondents indicated that "the program should remain the same" $(n=51)$, "the number of walks should be increased" $(n=21)$, and that "walk leaders should remain attached to the program" $(n=10)$.

Qualitative data gathered from exit interviews provided further insight into the reasons for attrition. "Health problems" was given as a major reason for dropping out of the program. "We enjoyed it, both of us [referring to her husband]; it's just that I have this bad heel." Others who dropped out indicated that there were 
competing priorities such as work and travel commitments. "I enjoyed the walking program, but we go away for a few months over the Christmas holiday period." Some walkers found the time unsuitable: "It [the walk] was too early in the morning." Walking groups did meet early in the morning, between 6:30 and 7:30 a.m. for approximately $1 \mathrm{hr}$, while it was relatively cool and also to reduce interference with other planned activities.

\section{Discussion}

Prior research has documented a range of characteristics associated with attrition. This study found, however, that age (Chatfield et al., 2005; Lui \& Anthony, 1989; Van Beijsterveldt et al., 2002), relationship status (Garcia \& King, 1991), level of education (Lui \& Anthony, 1989; Jacomb et al., 2002; Van Beijsterveldt et al.), and gender (Rejeski, Brawley, Ettinger, Morgan, \& Thompson, 1997) were not significantly related to attrition. Our results concur with those of others that certain demographic variables tend to be unstable predictors of exercise adherence (Martin \& Sinden, 2001).

Several factors found to affect attrition in this study were consistent with previous research. SEIFA was identified as significant, with greater risk of attrition occurring in low- and medium-socioeconomic areas, which supported findings in the literature (Boyette et al., 2002; Dishman, 1991; Ford et al., 1991). Overweight was also significantly associated with attrition, similar to in previous studies (Dishman, 1991). It has been well documented that the greater the BMI, the greater the likelihood of being insufficiently active (Bauman et al., 2002; Bull, Milligan, Rosenberg, \& MacGowan, 2000). Past history of exercise participation has been found to influence exercise adherence among older adults (Boyette et al.). Participants in our study who had a poor history of physical activity were twice as likely to drop out.

As in previous studies (Martin \& Sinden, 2001; O'Brien Cousins, 2003; Resnick, 2001), walking self-efficacy appeared to affect exercise adherence. A person's mood might influence his or her perception of walking efficacy, with better overall mental health being linked to stronger self-efficacy (Resnick). This notion is particularly pertinent because a higher loneliness score was found to be associated with attrition.

Loneliness is subjective and is related to the perception of social situation, by which individuals assess the quality and quantity of their relationships. Individuals are often reluctant to acknowledge deficiencies in their relationships that lead to feeling lonely (Russell, 1996). Therefore, the UCLA loneliness score does not use the term loneliness in any of its questions. Instead, it subtly identifies individuals who perceive their situation to be less than satisfactory, by way of feeling alone or not connecting with others. Previous research has demonstrated the connection between adherence to health intervention and psychological states (Martin \& Sinden, 2001) such as depression (Rejeski et al., 1997) and decreased cognition (Chatfield et al., 2005). The concept that such individuals are not suited to the group environment warrants further consideration.

Two thirds (65\%) of the walkers completed the PALS program, and $35 \%$ were lost to attrition. This is a moderate, acceptable attrition rate when compared with 
other programs involving older persons (Dishman, 1994; McAuley, Courneya, Rudolph, \& Lox, 1994; Morey et al., 2003). Of those who dropped out, most of the attrition (77\% of the dropouts; and $27 \%$ of total sample) occurred within the first 3 months. Once again, this is an acceptable figure when compared with other studies that reported between 20\% (Warren-Findlow et al., 2003) and 36\% (Schmidt et al., 2000) attrition of the total sample in the initial 3 months. Only $23 \%$ of the attrition occurred after this period, suggesting that the initial 3 months were critical in terms of attrition prevention for older adults.

There are a number of factors that might have influenced the attrition rate. First, this study did not recruit through advertising, a method that tends to reach self-selecting, highly motivated volunteers (Mills et al., 2001). Instead, a multistage strategy with a known population base was used. It aimed to access a more representative sample, which in turn might lead to the recruitment of a less motivated and less self-selecting population. Second, because the recruits were less physically active, with perhaps limited exercise experience, this might have meant that they had unrealistic expectations of the program and its content (Warren-Findlow et al., 2003). Third, it has been suggested that early program dropout can be related to program details and the program's fit with participants' capabilities and expectations (Warren-Findlow et al.). This intervention tailored the program to suit older, insufficiently active adults, but perhaps such a goal is difficult to achieve with random selection to groups. Fourth, randomization might have influenced early dropouts-some participants indicated that they did not care whether they were allocated to the control or the walking group but really would have preferred to be controls.

The PALS intervention program was designed to address acknowledged barriers to physical activity, such as program suitability and instructor and peer support (Corti et al., 1995; Dishman, 1994; Warren-Findlow et al., 2003). Consideration was given to access and cost of the program (Souder, 1992) and to the aesthetics of the environment (Corti, Donovan, \& Holman, 1996) and its safety (Booth, Bauman, Owen, \& Gore, 1997). Qualitative data from exit interviews supported the suitability of the program. The main reasons cited for dropping out were health problems, competing priorities, and the walks being too early. The first two factors were not program related, and the walking time was set early in the morning so as not to interfere with other daily activities.

This study considered individual factors, program factors, and environmental factors that might have affected the attrition of participants from the physical activity intervention program. The environmental factors were not statistically significant. This is expected, however, because the neighborhoods selected for this program contained at least $11 \%$ of people over 65 years old, so these suburbs were likely to be more established, with sound infrastructure including parks suitable for the walk groups.

\section{Conclusion}

The PALS study, based on SCT, demonstrated the complex interplay of factors that influence attrition. Early identification of physically inactive participants who are from low-socioeconomic areas, have high BMI and loneliness scores, and have 
low walking self-efficacy will help control attrition. Several issues demand further investigation. For example, walking self-efficacy has been shown to affect exercise adoption and maintenance. Therefore, intervention programs should develop and nurture this characteristic to enable individuals to continue with the program. The concept of loneliness also requires more investigation, because being in a group, yet feeling unfulfilled and isolated, is potentially problematic. These individuals might require more encouragement, affirmation, and feedback. Elderly people are heterogeneous, so new strategies are needed to mobilize them.

\section{References}

Anderson, L., Schnohr, P., Schroll, M., \& Hein, H. (2000). All-cause mortality associated with physical activity during leisure time, work, sports, and cycling to work. Archives of Internal Medicine, 160(11), 1621-1628.

Australian Bureau of Statistics. (1996). Census of population and housing: Socio-economic indexes for areas (SEIFA). Information paper (2039.0). Canberra : Australian Government Publishing Service.

Australian Bureau of Statistics. (2001). Census of basic community profile. Canberra: Australian Government Publishing Service.

Australian Department of Health and Aged Care. (1999). National physical activity guidelines for Australians. Canberra: Australian Government Publishing Service.

Bandura, A. (1986). Social foundations of thought and action. Englewood Cliffs, NJ: Prentice Hall.

Bandura, A. (1997). Self efficacy: The exercise of control. New York: Freeman.

Bauman, A., Bellew, B., Vita, P., Brown, W., \& Owen, N. (2002). Getting Australia active: Towards better practice for the promotion of physical activity. Melbourne, Australia: National Public Health Partnership.

Bauman, A., Smith, B., Stoker, L., \& Booth, M. (1999). Geographical influences upon physical activity participation: Evidence of a "coastal effect." Australian and New Zealand Journal of Public Health, 23(3), 322-324.

Booth, M., Bauman, A., Owen, N., \& Gore, C. (1997). Physical activity preferences, preferred sources of assistance, and perceived barriers to increased activity among physically inactive Australians. Preventive Medicine, 26, 131-137.

Boyette, L., Lloyd, A., Boyette, J., Watkins, E., Furbush, L., Dunbar, S., et al. (2002). Personal characteristics that influence exercise behaviour of older adults. Journal of Rehabilitation and Research and Development, 39(1), 95-103.

Bull, F., Milligan, R., Rosenberg, M., \& MacGowan, H. (2000). Physical activity levels of Western Australian adults 1999. Perth: Health Department of Western Australia, Western Australian Government.

Cameron, A., Welborn, T., Zimmet, P., Dunstan, D.W., Owen, N., Salmon, J., et al. (2003). Overweight and obesity in Australia: The 1999-2000 Australian diabetes, obesity and lifestyle study (AusDiab). Medical Journal of Australia, 178(9), 427-432.

Chatfield, M., Brayne, C., \& Matthews, F. (2005). A systematic literature review of attrition between waves in longitudinal studies in the elderly shows a consistent pattern of dropout between differing studies. Journal of Clinical Epidemiology, 58, 13-19.

Chogahara, M., O’Brien Cousins, S., \& Wankel, L. (1998). Social influences on physical activity in older adults: A review. Journal of Aging and Physical Activity, 6, 1-17.

Clark, D. (1999). Identifying psychological, physiological, and environmental barriers and facilitators to exercise among older low income adults. Journal of Clinical Geropsychology, 5(1), 51-62. 
Corti, B., Donovan, R., Castine, R., D’Arcy, C., Holman, J., \& Shilton, T. (1995). Encouraging the sedentary to be active everyday: Qualitative formative research. Health Promotion Journal of Australia, 5(2), 10-17.

Corti, B., Donovan, R., \& Holman, C. (1996). Factors influencing the use of physical activity facilities: Results from qualitative research. Health Promotion Journal of Australia, 6(1), 16-21.

Craig, C., Marshall, A., Sjostrom, M., Bauman, A., Booth, M., Ainsworth, B., et al. (2003). International physical activity questionnaire: 12 country reliability and validity. Medicine and Science in Sports and Exercise, *, 1381-1390.

Crespo, C., Palmieri, M., Perdomo, R., McGee, D., Smit, E., Sempos, C., et al. (2002). The relationship of physical activity and body weight with all-cause mortality: Results from the Puerto Rico Heart Health Program. Annals of Epidemiology, 12, 543-552.

Dishman, R. (1991). Increasing and maintaining physical activity and exercise. Behavioural Therapy, 41, 3-15.

Dishman, R. (1994). Motivating older adults to exercise. Southern Medical Journal, 87(5), S79-S82.

Ettinger, W., Burns, R., Messier, S., Applegate, W., Rejeski, W., Morgan, T., et al. (1997). A randomized trial comparing aerobic exercise and resistance exercise with a health education program in older adults with knee osteoarthritis. Journal of the American Medical Association, 277, 25-31.

Ford, E.S., Merritt, R.K., Heath, G.W., Powell, K.E., Washburn, R.A., Kriska, A., \& Haile, G. (1991). Physical activity behaviors in lower and higher socioeconomic status populations. American Journal of Epidemiology, 133, 1246-1256.

Garcia, A., \& King, A. (1991). Predicting long-term adherence to aerobic exercise: A comparison of two models. Journal of Sport \& Exercise Psychology, 13, 394-410.

Godin, G., Desharnais, R., Valois, P., Jobin, J., \& Bradet, R. (1994). Differences in perceived barriers to exercise between high and low intenders: Observations among different populations. American Journal of Health Promotion, 8(4), 279-285.

Hayslip, B., Weigand, D., Weinberg, R., Richardson, P., \& Jackson, A. (1996). The development of new scales for assessing health belief model constructs in adulthood. Journal of Aging and Physical Activity, 4(4), 307-323.

Jacomb, P., Jorm, A., Korten, A., Christensen, H., \& Henderson, A. (2002). Predictors of refusal to participate: A longitudinal health survey of the elderly in Australia. BMC Pubic Health, 2(4):

King, A., Blair, S., Bild, D., Dishman, R., Dubbert, P., Marcus, B., et al. (1992). Determinants of physical activity and interventions in adults. Medicine and Science in Sports and Exercise, 26(6), S221-S236.

King, A., Rejeski, W., \& Buchner, D. (1998). Physical activity interventions targeting older adults: A critical review and recommendations. American Journal of Preventive Medicine, 15(4), 316-333.

Lee, I., \& Skerrett, P. (2000). Physical activity and all-cause mortality: What is the doseresponse relation? Medicine and Science in Sports and Exercise, 33(6), s459-s471.

Lui, I., \& Anthony, J. (1989). Using the "Mini-Mental State" examination to predict elderly subjects' completion of a follow-up interview. American Journal of Epidemiology, 130, 416-422.

Martin, K., \& Sinden, A. (2001). Who will stay and who will go? A review of older adults' adherence to randomized controlled trials of exercise. Journal of Aging and Physical Activity, 9(2), 91-114.

McAuley, E., Courneya, K., Rudolph, L., \& Lox, C. (1994). Enhancing exercise adherence in middle-aged males and females. Gerontologist, 23(4), 498-506.

McAuley, E., Jerome, G., Elavsky, S., Marquez, D., \& Ramsey, S. (2003). Predicting long term maintenance of physical activity in older adults. Preventive Medicine, 37, 110-118. 
McAuley, E., \& Mihalko, S. (2003). Measuring exercise-related self-efficacy. In J. Duda (Ed.), Advances in sport and exercise psychology measurement (pp. 371-390). Morgantown, WV: Fitness Information Technology.

Mihalko, S., \& McAuley, E. (1996). Strength training effects on subjective well-being and physical function in the elderly. Journal of Aging and Physical Activity, 4, 56-68.

Mills, K., Stewart, A., McLellan, B., Verboncoeur, C., King, A., \& Brown, B. (2001). Evaluation of enrollment bias in a physical-activity-promotion program for seniors. Journal of Aging and Physical Activity, 9(4), 398-314.

Morey, M., Dubbert, P., Doyle, M., MacAller, H., Crowley, G., Kuchibhatla, M., et al. (2003). From supervised to unsupervised exercise: Factors associated with exercise adherence. Journal of Aging and Physical Activity, 11(3), 351-368.

O'Brien Cousins, S. (2003). Grounding theory in self-referent thinking: Conceptualizing motivation for older adult physical activity. Psychology of Sport and Exercise, 4, 81100.

Preloran, H., Browner, C., \& Lieber, E. (2001). Strategies for motivating Latino couples' participation in qualitative health research and their effects on sample construction. American Journal of Public Health, 91(11), 1832-1841.

Prohaska, T., Peters, K., \& Warren, J. (2000). Sources of attrition in a church-based exercise program for older African-Americans. American Journal of Health Promotion, 14(6), 380-385.

Rejeski, W., Brawley, L., Ettinger, W., Morgan, T., \& Thompson, C. (1997). Compliance to exercise therapy in older participants with knee osteoarthritis: Implications for treating disability. Medicine and Science in Sports and Exercise, 29, 977-985.

Resnick, B. (2001). Testing a model of exercise behaviour in older adults. Research in Nursing and Health, 24, 83-92.

Resnick, B., Palmer, M., Jenkins, L., \& Spellbring, A. (2000). Efficacy expectations and exercise behavior in older adults: A path analysis. Journal of Advanced Nursing, 32, 16-23.

Resnick, B., \& Spellbring, A. (2000). Understanding what motivates older adults to exercise. Journal of Gerontological Nursing, 26(3), 34-42.

Russell, D. (1996). UCLA loneliness scale (version 3): Reliability, validity, and factor structure. Journal of Personality Assessment, 66(1), 20-40.

Sallis, J.F., Bauman, A., \& Pratt, M.P. (1998). Environment and policy interventions to promote physical activity. American Journal of Preventive Medicine, 15(4), 379-397.

Schmidt, J., Gruman, C., King, M., \& Wolfson, L. (2000). Attrition in an exercise intervention: A comparison of early and later dropouts. Journal of the American Geriatrics Society, 48(8), 952-960.

Souder, J. (1992). The consumer approach to recruitment of elder subjects. Nursing Research, 41(5), 314-316.

Stahl, T., Rutten, A., Nutbeam, D., Bauman, A., Kannas, L., Abel, T., et al. (2001). The importance of the social environment for physically active lifestyle-Results from an international study. Science and Social Medicine, 52, 1-10.

Treasure, D., \& Newberry, D. (1998). Relationship between self-efficacy, exercise intensity, and feeling states in a sedentary population during and following an acute bout of exercise. Journal of Sport \& Exercise Psychology, 20, 1-11.

Van Beijsterveldt, C., Van Boxtel, M., Bosma, H., Houx, P., Buntinx, F., \& Jolles, J. (2002). Predictors of attrition in a longitudinal cognitive aging study: The Maastricht Aging Study (MAAS). Journal of Clinical Epidemiology, 55, 216-223.

Wannamethee, S., Shaper, A., \& Walker, M. (2000). Physical activity and mortality in older men with diagnosed coronary heart disease. Circulation, 102(12), 1358-1363.

Wanzhu, T., Stump, T.E., Damush, T.M., \& Clark, D. (2004). The effects of health and environment on exercise-class participation in older, urban women. Journal of Aging and Physical Activity, 12, 480-496. 
Warren-Findlow, J., Prohaska, T., \& Freedman, D. (2003). Challenges and opportunities in recruiting and retaining underrepresented populations into health promotion research. Gerontologist, 43, 37-46.

Wilson, A., \& Webber, I. (1976). Attrition in a longitudinal study of an aged population. Experimental Aging Research, 2(5), 367-387.

\section{Appendix A: Relevant Questions Asked at the Exit Interview}

- During the program you stopped attending-Was there any particular reason for this?

- How did you feel when you did not attend the program?

- Do you think you were encouraged?

- Was there anything else that could have been done to make you stay with the walking program?

- Can you think of any way we could have changed or improved the program to make it more appealing?

- What do you think would be a good or appropriate physical activity program? 